"Modular Multilevel Converter in Electrified Railway Systems: Applications of Rail Static Frequency Converters and Rail Power Conditioners"

International Young Engineers Forum (YEF-ECE) 2020, Costa da Caparica, Portugal

To cite:

M. Tanta, L. A. M. Barros, J. G. Pinto, A. P. Martins and J. L. Afonso, "Modular Multilevel Converter in Electrified Railway Systems: Applications of Rail Static Frequency Converters and Rail Power Conditioners," 2020 International Young Engineers Forum (YEFECE), Costa da Caparica, Portugal, 2020, pp. 55-60, doi: 10.1109/YEF-ECE49388.2020.9171814. 


\title{
Modular Multilevel Converter in Electrified Railway Systems: Applications of Rail Static Frequency Converters and Rail Power Conditioners
}

\author{
Mohamed Tanta $^{1}$, Luis A. M. Barros ${ }^{1}$, J. G. Pinto ${ }^{1}$, António P. Martins ${ }^{2}$, and João L. Afonso ${ }^{1}$ \\ ${ }^{1}$ Centro ALGORITMI - University of Minho, Guimarães - Portugal \\ ${ }^{2}$ SYSTEC Research Center - University of Porto, Porto - Portugal \\ ${ }^{1}\{$ mtanta | lbarros | gpinto |jla\}@dei.uminho.pt, 2ajm@fe.up.pt
}

\begin{abstract}
The Modular Multilevel Converter (MMC) has become the most preferable multilevel converter topology for medium and high-power systems, due to the MMC's high reliability and redundancy. This paper focuses on MMC applications in electrified railway traction grids, namely, applications of rail static frequency converters and rail power conditioners. These applications provide a higher railway traction grid capacity and improve the power quality of the three-phase power grid. Simulation results of a case study are presented to show the features when using the MMC in electrified railway grids.
\end{abstract}

Keywords-Modular Multilevel Converter (MMC), Rail Power Conditioner (RPC), Static Frequency Converter (SFC), Power Quality, Electrified Railway Systems.

\section{INTRODUCTION}

In the last decades, electrified railway traction grids have observed rapid growth due to the increased global demand for rail transportation, specifically, the passenger and the freight rail transport. However, nowadays, this fast development in the rail industry is a consequence of the vast advances made in the area of power electronics [1]. The potential applications of power electronics converters in the rail industry are enormous, for instance, integrating the renewable energy resources in the railway traction grids [2], using the train regenerative braking energy [3], [4], and overcoming the power quality deterioration of electrified railway traction grids [5]-[8].

Power quality has become a very important concept of power delivery, especially in the second-half of the 1990s. From the early use of electrified traction grids, the power quality improvement was a significant concern for railway operators. Then, establishing newly electrified railway lines was not so attractive for the transmission lines operators. The electrified railway lines introduce harmonics and cause a three-phase power imbalance and negative sequence components (NSC) of currents, affecting the power quality of the three-phase power grid. In this context, power quality improvement by using power converters is indispensable, which leads to an increase in the electrified traction grid capacity, besides reducing the running costs of the trains [6].

Modular multilevel converter (MMC) was first introduced in 2001 by Marquardt Group for medium-voltage applications [9]. The topology itself was first used for medium-voltage transmission lines because of the scalability and the low harmonic distortion in the output waveform [10]. After 15 years of evolution and improvement, the MMC has become the most preferable multilevel converter topology for the medium and high-power systems despite the need for reactors to suppress the circulating currents between the MMC legs [11]. In this paper, MMC applications in electrified railway traction grids are presented, namely, the rail static frequency converter (SFC) and the rail power conditioner (RPC) based on MMC.

SFC based on MMC solution is mainly used to interface between the three-phase power grid and the single-phase railway traction grid. It maintains a balanced three-phase grid currents and prevents the harmonic contents to pass from the single-phase railway traction grid to the three-phase power grid [10]. On the other hand, RPC based on MMC solution is normally installed at the single-phase railway traction grid. It compensates harmonic currents injected by the electric locomotives. In addition, it overcomes the three-phase currents imbalance by providing active power and compensating reactive power. Consequently, a unitary power factor is obtained at the three-phase power grid since the reactive power exchange is between the RPC and the single-phase railway traction grid [12].

The paper is organized as follows: Section II describes the MMC operation principle and Section III presents the solution of SFC based on MMC. Section IV presents the solution of RPC based on MMC as well as the simulation results of a case study. Finally, Section V summarizes the conclusion of the work.

\section{MOdular MultileVEl CONVERTER OPERATION PRINCIPLE}

Figure 1 presents the operation approach of the MMC. Three-phase MMC contains three legs that correspond to the three-phase AC power grid. Each phase leg consists of two arms, namely an upper arm and a lower arm. Each arm contains a few tens of cascade-connected submodules (SM). Each SM is an independent voltage-source converter with at least two power switches and one floating DC-link capacitor. The total DC-link voltages of SM in one MMC arm should be able to withstand the entire converter DC-link voltage $V_{d c}$. By other side, each leg must have a total DC-link voltage with a value double the entire MMC DC-link voltage. By adopting an appropriate switching strategy, each MMC arm voltage has a sinusoidal output waveform with a DC offset equal to half the entire DC-link voltage. Figure 1 depicts the operation approach of the 5-level MMC, besides a basic representation of the 
MMC. The AC output voltage, $u_{o}$, is acquired by inserting or bypassing a specific number of SM in each MMC leg [13].

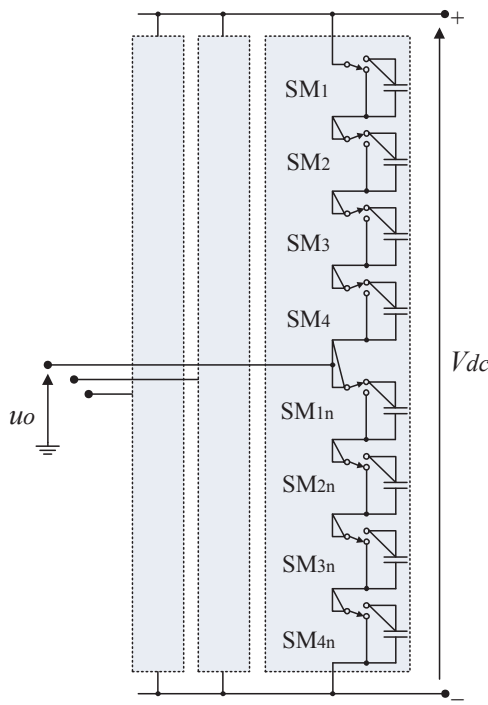

(a)

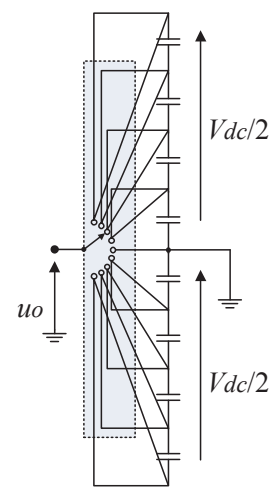

(b)
Figure 1. Operation approach the 5-level MMC: (a) Basic representation of the MMC; (b) Simplified representation of the MMC.

The DC-link voltage capacitor of each SM can be inserted (as the case of $\mathrm{SM}_{2}, \mathrm{SM}_{3}, \mathrm{SM}_{4}$, and $\mathrm{SM}_{1 \mathrm{n}}$ ) or bypassed (as the case of $\mathrm{SM}_{1}, \mathrm{SM}_{2 \mathrm{n}}, \mathrm{SM}_{3 \mathrm{n}}$, and $\mathrm{SM}_{4 \mathrm{n}}$ ) as shown in Figure 1 (a). A higher number of DC-link capacitors signifies a higher output voltage level, lower harmonic distortions, and a lower noise to the neighboring electrical equipment [13]. The simplified representation of $\mathrm{MMC}$ can be equivalent to a switch that changes its position and connection points with charged floating capacitors during a time cycle as shown in Figure 1 (b). At that case, a multilevel output voltage, $u_{o}$, is generated.

MMC leg AC output voltage is obtained collaboratively through the upper and the lower MMC arms, after considering mid-neutral point capacitors at the DC-link, $V_{d c}$, to create a neutral point. Operation states of the 5-level MMC are presented in Figure 2. MMC is structurally scalable and can hypothetically meet any voltage level requirement. The voltage level at the midpoint of the phase leg, $u_{o}$, is defined by the number of SM that are connected in the upper and lower MMC arms. By considering 5-level MMC, State 1 happens when all the upper arm SM are inserted and the lower arm SM are bypassed. At that case, the output voltage, $u_{o}$, has the highest negative value. On the other hand, State 2 is when three SM in the upper arm and one SM in the lower arm are inserted. State 3 is when two SM in the upper arm and two SM in the lower arm are inserted and the output voltage, $u_{o}$, is near zero. State 4 is when one SM in the upper arm and three SM in the lower arm are inserted. State 5 is when the entire lower arm SMs are inserted and the upper arm SMs are bypassed. At that case, the output voltage, $u_{o}$, reach the highest positive value [14].

\section{RAIL STATIC FREQUENCY CONVERTER BASED ON MMC}

The main MMC advantage is to have a low actual switching frequency of power electronic switches [1]. Consequently, the equivalent switching frequency, in this case, is equal to the actual switching frequency for each SM multiplied by the number of SM connected in series in each arm (each leg consists of two arms as shown in Figure 3). This leads to lower
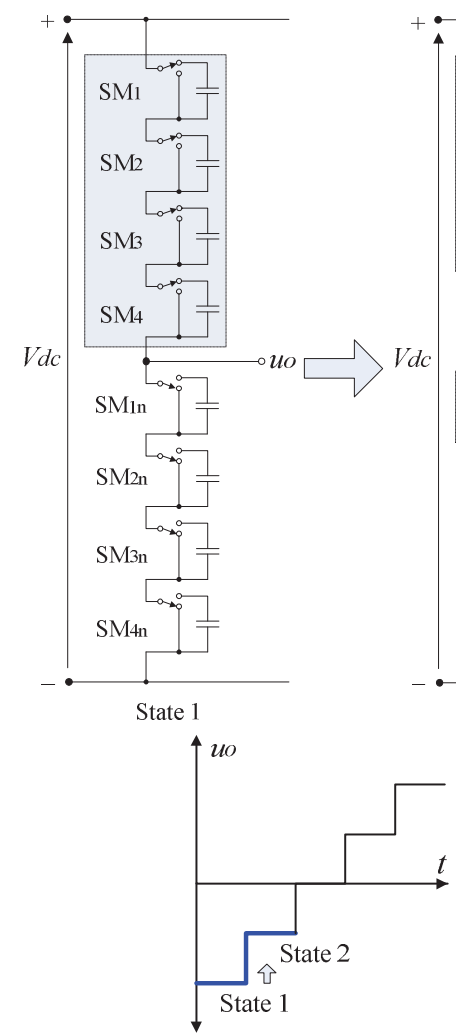
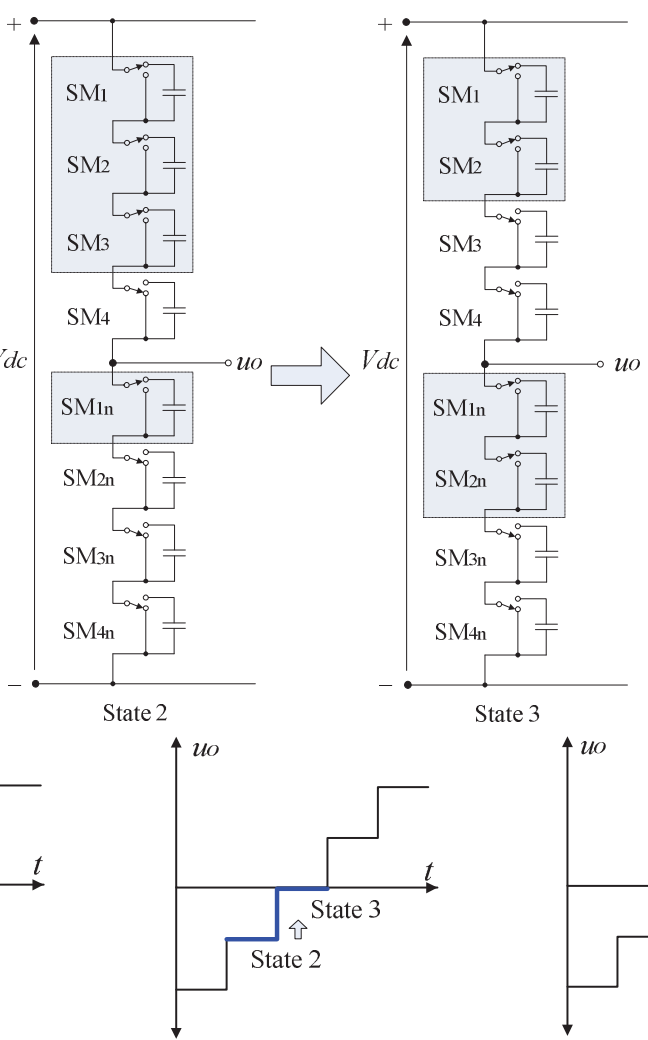
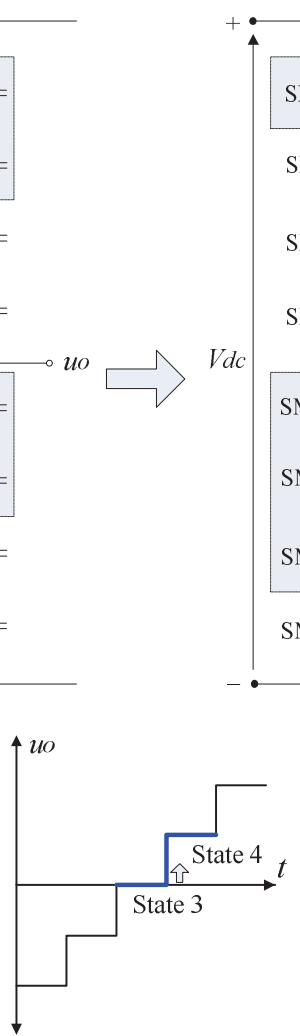
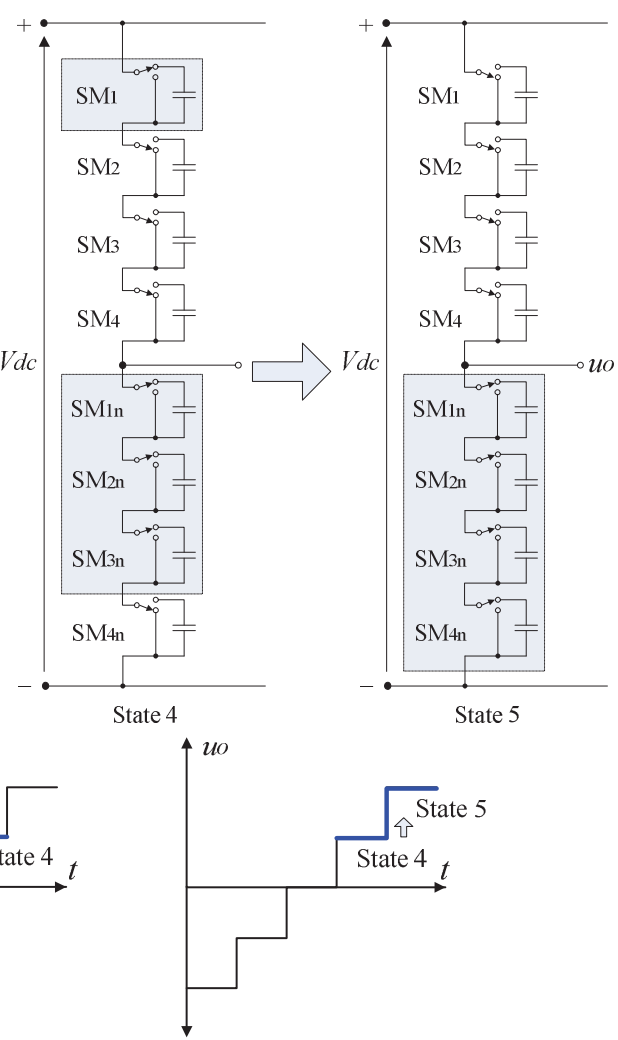

Figure 2. Operation states of the 5-level MMC: State 1 with 4 upper arm SM inserted; State 2 with 3 upper arm SM and 1 lower arm SM inserted; State 3 with 2 upper arm SM and 2 lower arm SM inserted; State 4 with 1 upper arm SM and 3 lower arm SM inserted; State 5 with 4 lower arm SM inserted. 
switching losses and a higher total efficiency. There is no need for the step-up power transformer to interface between the SFC based on MMC and the single-phase traction power grid, since the MMC can withstand the catenary medium-voltage of $25 \mathrm{kV}$ or $15 \mathrm{kV}$ [10]. This reduces the total costs, size, and the cooling equipment of the system. At present, the majority of commercial MMC projects are based on the AC/DC/AC indirect MMC with half-bridge SM. This is due to the high efficiency of the half-bridge SM, besides its low costs and the simple construction compared to other types of SM.

\section{A. SFC Based on Direct AC/AC MMC}

Direct MMC fulfills a direct three-phase to single-phase AC/AC conversion without DC-link connection. The main structure of direct $\mathrm{MMC}$ is composed of many identical series-connected bipolar full-bridge SM. Each SM consists of four power electronic modules (IGBT + free-wheeling Diodes) and a DC-link capacitor as shown in Figure 3. The direct MMC full-bridge SM allows the SM capacitor to be inserted into the circuit in any polarity. The MMC leg contains two arms and two reactors to smooth the current waveforms and to limit the circulating currents between the MMC arms. The number of series-connected SM in each arm can be defined according to the desired power level. Normally, in SFC railway substation, the number of SM in each arm is between 10 and 30 with a power level between 20 and $60 \mathrm{MVA}$. For example and for a catenary voltage of $15 \mathrm{kV}$, the peak voltage value is around $21 \mathrm{kV}$ could be obtained by seven SM per arm (14 SM per MMC leg) with DC-link capacitors each of $3.25 \mathrm{kV}$ [10]. Direct AC/AC MMC was discussed for the first time in [15].

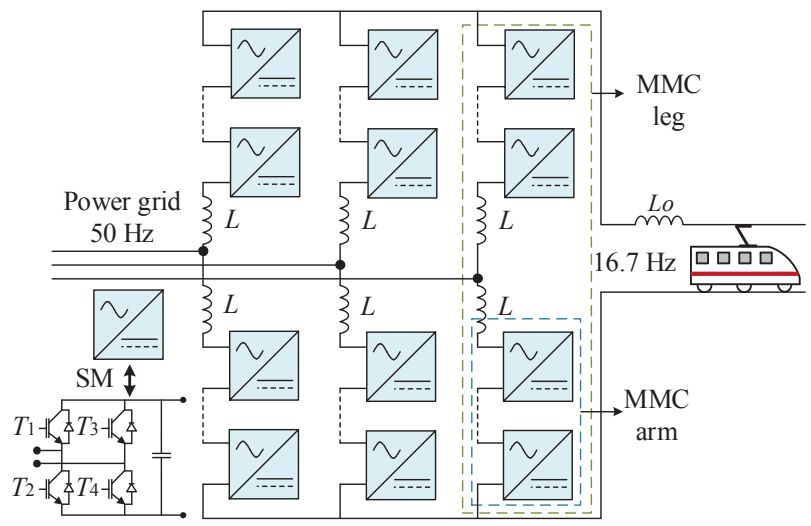

Figure 3. SFC based on direct AC/AC MMC.

Under normal operation, the direct AC/AC MMC converter circulates power between the three legs to compensate pulsating power on the catenary line. This circulated power has two frequencies, the sum and the difference between input and output power frequencies. If the input and the output frequencies are the same, so there is no circulating power between legs. This converter is convenient to be used for $16.7 \mathrm{~Hz}$ output frequency in railway substations because the difference in circulating power frequency between legs $(33.3 \mathrm{~Hz})$ is higher than the output frequency, thus this decreases the importance for filters requirement. Nevertheless, using direct MMC topology in practical applications is still limited and has some difficulties as it requires a frequency separation accompanied by a complex control as well.
Otherwise, the implementation will result in a harmonic interaction between the three-phase power grid and the single-phase traction power grid, especially at low numbers of $\mathrm{SM}$ and low switching frequency values [10]. Transformerless direct $\mathrm{MMC}$ is under interest for the interconnection between the $15 \mathrm{kV} / 16.7 \mathrm{~Hz}$ railway traction grid and the $50 \mathrm{~Hz}$ three-phase power grid, since this solution allows a direct connection without a $16.7 \mathrm{~Hz}$ bulky transformer [16].

Arm currents of the direct $\mathrm{AC} / \mathrm{AC} \mathrm{MMC}$ have mainly three different frequency components, the fundamental frequency current component of $50 \mathrm{~Hz}$, that has the main responsibility for power exchange between the direct $\mathrm{MMC}$ and the three-phase power grid, the $16.7 \mathrm{~Hz} \quad(50 / 3 \mathrm{~Hz})$ current component, that manages the power exchange between the direct MMC and the single-phase traction power grid, and the two-third of $(33 \mathrm{~Hz})$ fundamental frequency current component (the difference of input and output current frequencies) that controls the power exchange between the direct MMC arms (circulating current between arms). The last component refers to the circulating current flows between the MMC arms, which should be well controlled to avoid high power losses in the converter. The circulating current between arms can also be created from an additional current component that has the frequency of $(50+16.7 \mathrm{~Hz})$ (the sum of input and output current frequencies). Finally, current waveforms may also contain some additional harmonic contents. The main challenge in the direct MMC control system is the simultaneous currents control for the MMC legs in a way to generate AC current reference and also to maintain equal DC-link voltages among the MMC SM [10].

\section{B. SFC Based on Indirect AC/DC/AC MMC}

This MMC topology comprises series-connected half-bridge SM as shown in Figure 4. Each half-bridge SM contains two power electronic modules (IGBT + free-wheeling Diodes) and a DC-link capacitor. Besides the SM DC-links, there is a main DC-link connection between the three-phase $\mathrm{AC} / \mathrm{DC}$ converter and the single-phase DC/AC converter. Although the indirect $\mathrm{MMC}$ can accomplish a frequency conversion. However, this topology is used when the input frequency and the output frequency are the same since there is no limitation on frequency separation [10]. Concerning the railway applications, indirect $\mathrm{MMC}$ is the best choice for $50 \mathrm{~Hz}$ catenary supply [10]. On the other side and for $16.7 \mathrm{~Hz}$ catenary supply, indirect MMC should be used with larger SM capacitors and higher current rating devices on its single-phase $\mathrm{DC} / \mathrm{AC}$ converter compared to the direct MMC topology [16].

In the indirect $\mathrm{MMC}$, each $\mathrm{SM}$ acts as an independent half-bridge two-level converter generating a voltage of either 0 or $V_{d c}(\mathrm{SM}$ capacitor voltage). Using enough number of series-connected SM increases the converter power capacity. In addition, this makes the power electronic modules synthesize a stepped voltage that varies close to a sine waveform with very low harmonic contents [1]. As a conclusion, SFC solutions have the advantage of accomplishing direct frequency conversion, three-phase grid power factor correction, and reducing the number of neutral sections electrified railway traction grid. However, SFC overloading capability is a weakness point [6], [10]. 


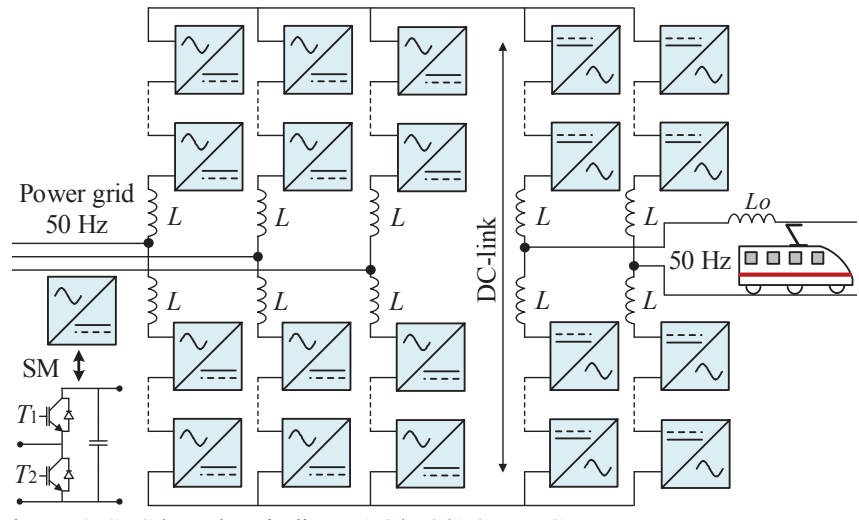

Figure 4. SFC based on indirect AC/DC/AC MMC.

\section{RAIL POWER CONDITIONER BASED ON INDIRECT AC/DC/AC MMC AND V/V POWER TRANSFORMER}

\section{A. RPC Based on Indirect MMC Topology}

RPC based on MMC can be considered as a three-phase MMC operating to compensate the NSC of currents, reactive power, and harmonics. RPC based on MMC compensates reactive power and shifts half of the active power difference between the load sections [12]. On the other hand, this solution does not require step-down coupling transformers or isolation transformers to interface between the RPC based on MMC and the single-phase traction power grid. The RPC based on an indirect $\mathrm{MMC}$ is shown in Figure 5. Each half-bridge SM contains two power switches and one capacitor which could be inserted or bypassed. Due to the V/V connection, the two load sections instantaneous voltages, $u_{x}$, and $u_{y}$ are 60 electrical degrees out of phase. A comparative study of different RPC topologies in [17], proved that the RPC based on the three-legs indirect MMC shows the superior performance in the $\mathrm{V} / \mathrm{V}$ traction system. Therefore, this paper presents the simulation results of the RPC based on three-legs indirect MMC.

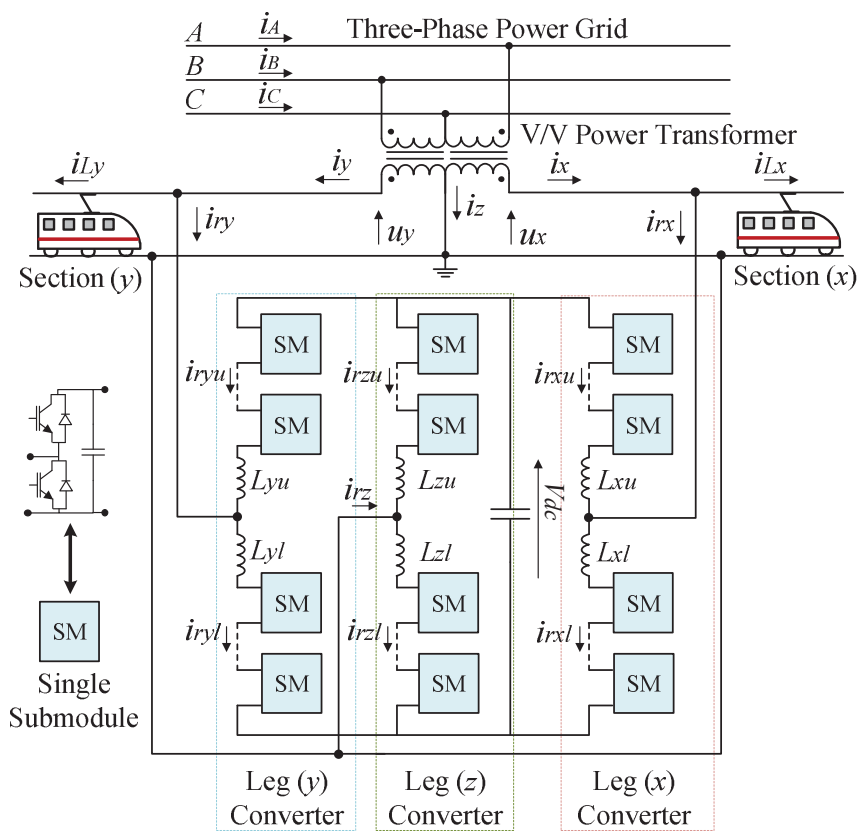

Figure 5. RPC based on indirect AC/DC/AC MMC.

\section{B. Simulation Results: Case Study}

The simulation model of RPC based on MMC and the V/V power transformer has been built by using the PSIM software for power electronics simulation. The simulation model consists of a 7-level $(N=7)$ MMC with a total number of $36 \mathrm{SM}$. The main parameters of the simulation model are presented in Table 1.

Load parameters presented in Table 2 are used in this simulation after considering a unitary load power factor for both load sections. Therefore, the locomotives can be modeled as a resistive load connected in parallel with an uncontrolled full bridge rectifier on the secondary side of the locomotive transformer. This full bridge rectifier is considered as a harmonic source, where the full locomotive model is shown in Figure 6 . The simulation model assumes that both load sections are unequally loaded. In this study, the higher load is at phase $x$ catenary, which has a value of $4.8 \mathrm{MW}$ and phase $y$ catenary is loaded with a value of $2.4 \mathrm{MW}$ as presented in Table 2 .

Table 1. Simulation parameters for the RPC based on MMC.

\begin{tabular}{lll}
\hline \hline Parameter & Symbol & Value \\
\hline \hline Line-to-line three-phase & $U_{A B}, U_{B C}$, & $220 \mathrm{kV}$ \\
voltage & $U_{C A}$ & $25 \mathrm{kV}$ \\
Catenary voltage & $U_{x}, U_{y}$ & $2.8 \mathrm{mH}$ \\
RPC buffer inductance & $L_{\sigma u}, L_{\sigma n u}, L_{\sigma l}$, & \\
& $L_{\sigma n l,}$ & \\
Catenary lumped & $Z_{L}$ & $0.05+\mathrm{j} 0.15 \Omega$ \\
impedance & $C_{S M}$ & $500 \mu \mathrm{F}$ \\
SM capacitor & $C_{d c}$ & $4 \mathrm{mF}$ \\
Main DC-link & $f_{i s w}$ & $3 \mathrm{kHz}$ \\
capacitance & $V_{S M}$ & $8.3 \mathrm{kV}$ \\
SM Switching frequency & $50 \mathrm{kV}$ \\
SM voltage & $V_{d c}$ & 6 \\
DC-link voltage & $N-1$ & \\
Number of SM in each & & \\
arm & &
\end{tabular}

Table 2. Load parameters used in the simulation of the RPC based on MMC.

\begin{tabular}{lll}
\hline \hline Symbol & Section $x$ & Section $y$ \\
\hline \hline$R_{1}$ & $0.8 \Omega$ & $1.6 \Omega$ \\
$R_{2}$ & $1 \Omega$ & $2 \Omega$ \\
$L_{2}$ & $2 \mathrm{mH}$ & $4 \mathrm{mH}$ \\
$P_{L}$ & $4.8 \mathrm{MW}$ & $2.4 \mathrm{MW}$ \\
\hline \hline
\end{tabular}

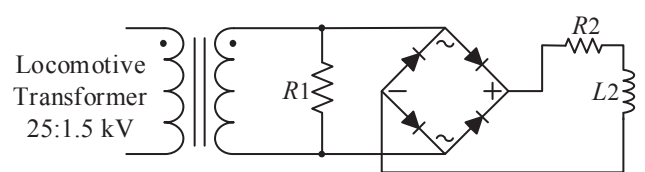

Figure 6. Locomotive equivalent circuit in the simulation model.

The three-phase power grid currents before applying the compensation strategy are presented in Figure 7 (a) and they are imbalanced currents. The currents after compensating reactive power and shifting half of the active power difference from the highly to the lightly loaded section are presented in Figure 7 (b). Figure 7 (c) shows the currents on the secondary side of the $\mathrm{V} / \mathrm{V}$ power transformer after compensation. These 
currents have $120^{\circ}$ out of phase and sinusoidal waveforms (after compensation) instead of $60^{\circ}$ out of phase (before compensation). It is noteworthy to mention, the used power transformers in the simulation model are considered as ideal ones without considering leakage flux, copper losses and core losses. In other words, the ideal transformer gives an output power exactly equal to its input power.

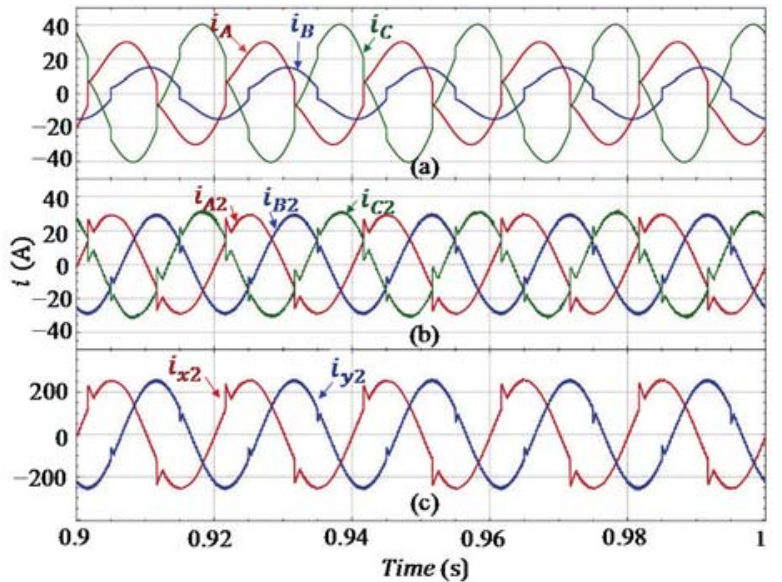

Figure 7. RPC based on MMC simulation results: (a) Three-phase currents before compensation; (b) Three-phase currents after compensation; (c) Currents at the secondary side of the V/V power transformer after compensation (when both load sections are loaded).

The load section currents are presented in Figure 8 (a), where $i_{L x}$ has double the value of $i_{L y}$. Both currents are considered as harmonic sources because of using an uncontrolled rectifier in the locomotive model. The compensation currents synthesized by the RPC are demonstrated in Figure 8 (b). The total instantaneous value of $i_{r x}+i_{r y}+i_{r z}$ at any time instant should be equal to zero.

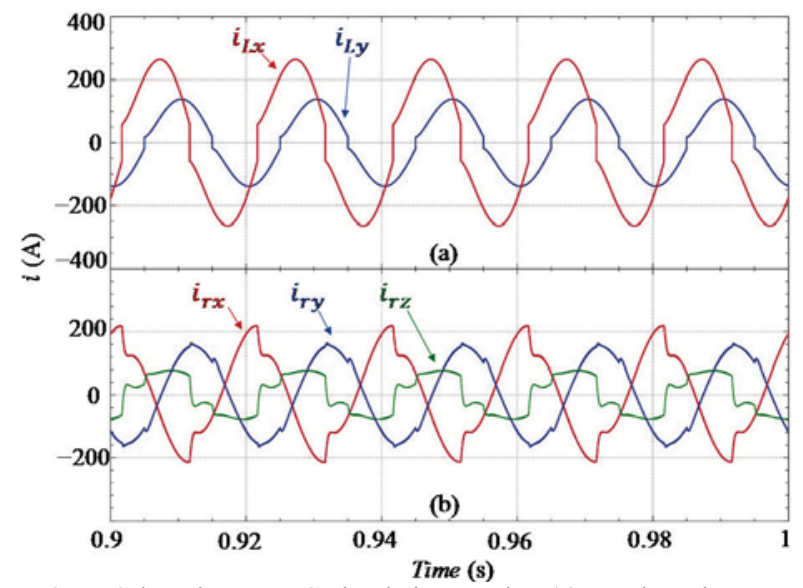

Figure 8. RPC based on MMC simulation results: (a) Load section currents; (b) Compensation currents.

Compensation currents are shared among the upper and the lower arms of the converter, then, currents of the upper arms, $i_{r x u}, i_{r y u}, i_{r z u}$, besides currents of the lower arms $i_{r x l}, i_{r y l}, i_{r z l}$ are presented in Figure 9. Apparently, the DC current component, the fundamental frequency current component of $50 \mathrm{~Hz}$, and the double fundamental frequency current component of $100 \mathrm{~Hz}$ exist in all arm currents, as shown in the frequency spectrum presented in Figure 10.

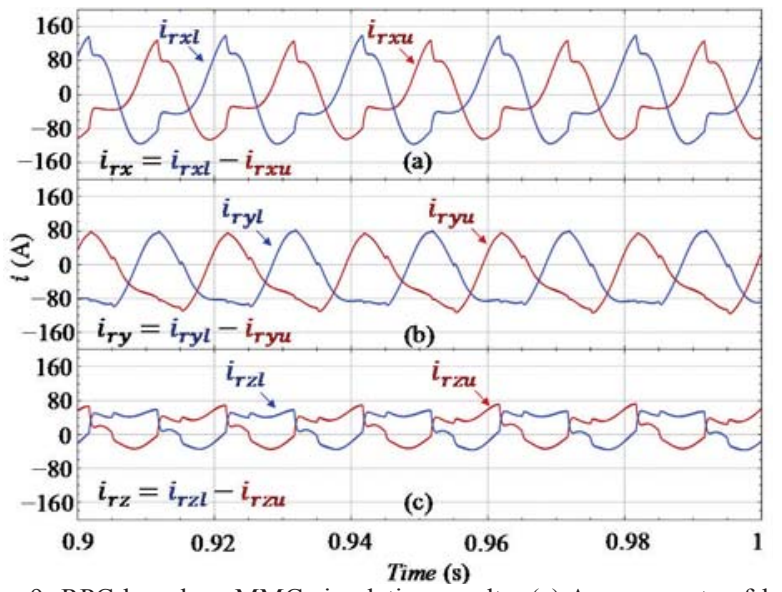

Figure 9. RPC based on MMC simulation results: (a) Arm currents of leg $x$; (b) Arm currents of leg $y$; (c) Arm currents of leg $z$.

The DC component is mainly responsible for the DC power transference between the converter legs and the main DC-link (charging and discharging of the SM capacitors), while the fundamental frequency component forms the compensation currents synthesized by the converter. The double fundamental frequency component signifies the circulating current between the MMC arms. Obviously, and as shown in Figure 10 (a), the compensation currents do not contain any DC components or double fundamental frequency components. By another meaning, these currents do not contribute to the compensation currents synthesized by the converter. All currents presented in Figure 10 have harmonic contents of the third, fifth, etc., order. However, high order harmonic contents are normally neglected since they have small values.

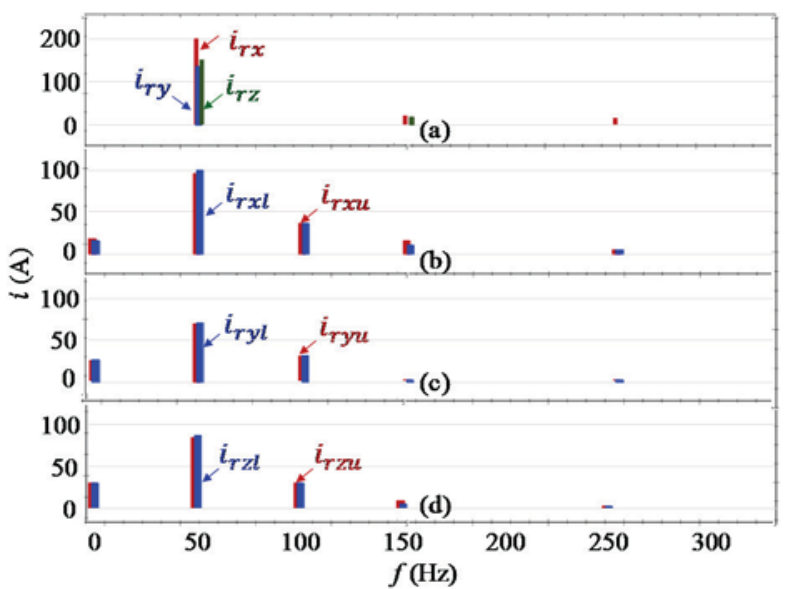

Figure 10. RPC based on MMC frequency spectrum simulation results: (a) Compensation currents; (b) Arm currents of leg $x$; (c) Arm currents of leg $y$; (d) Arm currents of $\operatorname{leg} z$.

Figure 11 shows the results of the MMC SM voltages. All $\mathrm{SM}$ voltages are near a reference value of $8.3 \mathrm{kV}$. Each half-bridge SM voltage has a voltage ripple frequency equal to the fundamental frequency of $50 \mathrm{~Hz}$ as shown in Figure 11 (a), Figure 11 (b) and Figure 11 (c). However, the voltage ripple frequency of the main DC-link is the double of the fundamental frequency as shown in Figure 11 (d). The main disadvantage of the half-bridge SM is the low voltage ripple frequency, which has a value equal to the fundamental frequency. This may imply to use bulky SM capacitors. 


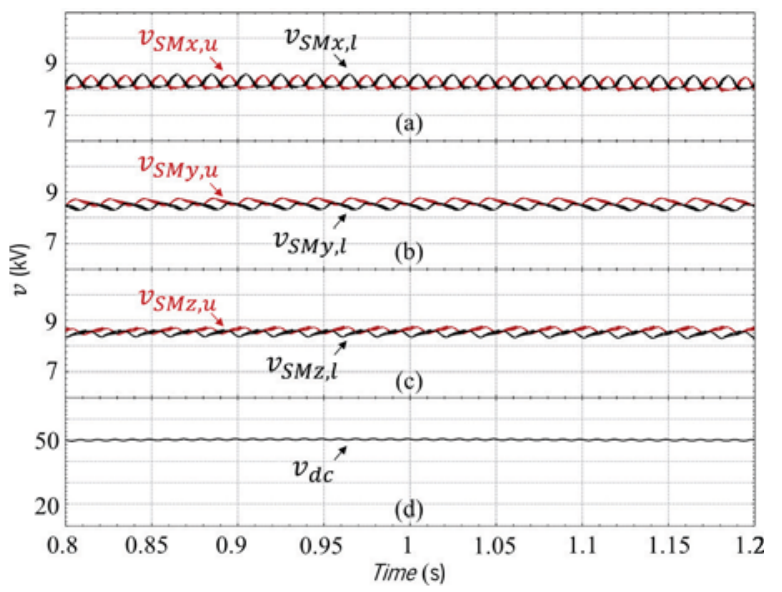

Figure 11. RPC based on MMC SM voltages simulation results: (a) Leg $x$; (b) Leg $y$; (c) Leg $z$; (d) DC-link voltage.

\section{CONCLUSION}

The modular multilevel converter (MMC) is an emerging transformerless solution that offers output waveforms with high power quality. It is a scalable solution that has the capability to meet any voltage level with superior harmonic performance, and reduced values of the converter components rating. This paper presented the MMC topology and its applications in the area of electric railway transportation. The MMC operation principle, besides two main applications of the MMC in this area, have also been introduced. The Static Frequency Converter (SFC) based on MMC topology has been discussed, showing the main technical considerations when using this solution in different railway traction power grids, that have dissimilar catenary voltage and frequency $(15 \mathrm{kV} / 16.7 \mathrm{~Hz}$ and $25 \mathrm{kV} / 50 \mathrm{~Hz}$ ). The SFC solution improves the three-phase grid power quality and maintains a unitary power factor. On the other hand, this paper discussed the application of rail power conditioner (RPC) based on MMC, where such a solution compensates reactive power, harmonics, and overcomes the imbalance in the three-phase power grid. Computer simulations for one of these applications have been established, namely, the RPC based on MMC topology. Simulation results confirmed the effectiveness of the RPC based on MMC solution for the purpose of power quality improvement. It can be concluded that the MMC is a viable solution for future railway transportation applications.

\section{ACKNOWLEDGEMENT}

This work has been supported by the Portuguese Foundation of Science and Technology (FCT), within the R\&D Units Project Scope: UIDB/00319/2020 and PTDC/EEIEEE/28813/2017. The first author Mohamed Tanta is supported by FCT Ph.D. grant with a reference $\mathrm{PD} / \mathrm{BD} / 127815 / 2016$.

\section{REFERENCES}

[1] D. Ronanki and S. S. Williamson, "Modular Multilevel Converters for Transportation Electrification: Challenges and Opportunities," IEEE Trans. Transp. Electrification, vol. 4, no. 2, pp. 399-407, Jun. 2018, doi: 10.1109/TTE.2018.2792330.
[2] İ. Şengör, H. C. Kılıçkıran, H. Akdemir, B. Kekezo\&gcaron;lu, O. Erdinç, and J. P. S. Catalão, "Energy Management of a Smart Railway Station Considering Regenerative Braking and Stochastic Behaviour of ESS and PV Generation," IEEE Trans. Sustain. Energy, vol. 9, no. 3, pp. 1041-1050, Jul. 2018, doi: 10.1109/TSTE.2017.2759105.

[3] G. Cui et al., "Supercapacitor Integrated Railway Static Power Conditioner for Regenerative Braking Energy Recycling and Power Quality Improvement of High-Speed Railway System," IEEE Trans. Transp. Electrification, vol. 5, no. 3, pp. 702-714, Sep. 2019, doi: 10.1109/TTE.2019.2936686.

[4] K. Aoki, K. Kikuchi, M. Seya, and T. Kato, "Power Interchange System for Reuse of Regenerative Electric Power," Hitachi Rev., vol. 67, no. 7, pp. 71-75, 2018.

[5] D. Serrano-Jiménez, L. Abrahamsson, S. Castaño-Solís, and J. SanzFeito, "Electrical railway power supply systems: Current situation and future trends," Int. J. Electr. Power Energy Syst., vol. 92, pp. 181-192, Nov. 2017, doi: 10.1016/j.ijepes.2017.05.008.

[6] M. Tanta, V. Monteiro, T. J. C. Sousa, A. P. Martins, A. S. Carvalho, and J. L. Afonso, "Power quality Phenomena in Electrified Railways: Conventional and New Trends in Power Quality Improvement toward Public Power Systems," in 2018 International Young Engineers Forum (YEF-ECE), Costa da Caparica, May 2018, pp. 25-30, doi: 10.1109/YEF-ECE.2018.8368934.

[7] J. G. Pinto, M. Tanta, V. Monteiro, L. A. M. Barros, and Joao. L. Afonso, "Active Power Conditioner Based on a Voltage Source Converter for Harmonics and Negative Sequence Components Compensation in Electrified Railway Systems," presented at the Transport Research Arena TRA 2018, Vienna, Austria, Apr. 2018, pp. 1-10, doi: 10.5281/zenodo.1491309.

[8] M. Tanta, J. G. Pinto, V. Monteiro, A. P. Martins, A. S. Carvalho, and J. L. Afonso, "Topologies and Operation Modes of Rail Power Conditioners in AC Traction Grids: Review and Comprehensive Comparison," Energies, vol. 13, no. 9, p. 2151, Jan. 2020, doi: 10.3390/en13092151.

[9] H. M. P. and M. T. Bina, "A Transformerless Medium-Voltage STATCOM Topology Based on Extended Modular Multilevel Converters," IEEE Trans. Power Electron., vol. 26, no. 5, pp. 1534-1545, May 2011, doi: 10.1109/TPEL.2010.2085088.

[10] I. Krastev, P. Tricoli, S. Hillmansen, and M. Chen, "Future of Electric Railways: Advanced Electrification Systems with Static Converters for AC Railways," IEEE Electrification Mag., vol. 4, no. 3, pp. 6-14, Sep. 2016, doi: 10.1109/MELE.2016.2584998.

[11] J. Moon, C. Kim, J. Park, D. Kang, and J. Kim, "Circulating Current Control in MMC Under the Unbalanced Voltage," IEEE Trans. Power Deliv. vol. 28, no. 3, pp. 1952-1959, Jul. 2013, doi: 10.1109/TPWRD.2013.2264496.

[12] M. Tanta et al., "Simplified Rail Power Conditioner Based on a HalfBridge Indirect $\mathrm{AC} / \mathrm{DC} / \mathrm{AC}$ Modular Multilevel Converter and a V/V Power Transformer," in IECON 2017 - 43rd Annual Conference of the IEEE Industrial Electronics Society, Beijing, China, Oct. 2017, pp. 6431-6436, doi: 10.1109/IECON.2017.8217120.

[13] A. Nami, J. Liang, F. Dijkhuizen, and G. D. Demetriades, "Modular Multilevel Converters for HVDC Applications: Review on Converter Cells and Functionalities," IEEE Trans. Power Electron., vol. 30, no. 1, pp. 18-36, Jan. 2015, doi: 10.1109/TPEL.2014.2327641.

[14] M. Ishfaq et al., "A New Adaptive Approach to Control Circulating and Output Current of Modular Multilevel Converter," Energies, vol. 12, no. 6, p. 1118, Jan. 2019, doi: 10.3390/en12061118.

[15] R. W. Erickson and O. A. Al-Naseem, "A new family of matrix converters," in IECON'01. 27th Annual Conference of the IEEE Industrial Electronics Society (Cat. No.37243), Nov. 2001, vol. 2, pp. 1515-1520 vol.2, doi: 10.1109/IECON.2001.976015.

[16] M. Winkelnkemper, A. Korn, and P. Steimer, "A Modular Direct Converter for Transformerless Rail Interties," in 2010 IEEE International Symposium on Industrial Electronics, Jul. 2010, pp. 562-567, doi: 10.1109/ISIE.2010.5637826.

[17] Q. Xu et al., "Analysis and Comparison of Modular Railway Power Conditioner for High-Speed Railway Traction System," IEEE Trans. Power Electron., vol. PP, no. 99, pp. 6031-6048, 2016, doi: 10.1109/TPEL.2016.2616721. 